

\title{
Multiple Scale Analysis for Divergence-Hopf Bifurcation of Imperfect Symmetric Systems
}

\author{
Angelo Luongo, Achille Paolone
}

\section{To cite this version:}

Angelo Luongo, Achille Paolone. Multiple Scale Analysis for Divergence-Hopf Bifurcation of Imperfect Symmetric Systems. Journal of Sound and Vibration, 1998, 218 (3), pp.527-539. hal-00812557

\section{HAL Id: hal-00812557 https://hal.science/hal-00812557}

Submitted on 12 Apr 2013

HAL is a multi-disciplinary open access archive for the deposit and dissemination of scientific research documents, whether they are published or not. The documents may come from teaching and research institutions in France or abroad, or from public or private research centers.
L'archive ouverte pluridisciplinaire HAL, est destinée au dépôt et à la diffusion de documents scientifiques de niveau recherche, publiés ou non, émanant des établissements d'enseignement et de recherche français ou étrangers, des laboratoires publics ou privés. 


\title{
MULTIPLE SCALE ANALYSIS FOR DIVERGENCE-HOPF BIFURCATION OF IMPERFECT SYMMETRIC SYSTEMS
}

\author{
A. Luongo and A. Paolone \\ Dipartimento di Ingegneria delle Strutture, delle Acque e del Terreno, \\ Università di L'Aquila, Monteluco di Roio, 67040 L'Aquila, Italy
}

\begin{abstract}
The multiple time-scale method is adapted to study the post-critical behavior of general non-conservative symmetric systems, possibly affected by imperfections, for which divergence and Hopf bifurcations interact. The procedure illustrated makes it possible to elude the computational burden related to the application of the center manifold reduction. It also furnishes explicit expressions of the coefficients of the standard normal form bifurcation equations in terms of the coefficients of the original system. As an example, the method is applied to a two-degree-of-freedom rigid bar subjected to axial load (Augusti's model) and transversal flow. The critical and post-critical scenarios are analyzed in detail, for both the perfect and imperfect systems.
\end{abstract}

\section{INTRODUCTION}

Mechanical systems under non-conservative excitation may exhibit different types of bifurcation and post-critical behavior, especially when interaction phenomena occur. An interesting case occurs when a divergence and a Hopf bifurcation manifest themselves at (nearly) coincident values of the parameters set [1]. Apart from particular cases, this bifurcation is structurally stable when at least two control parameters are considered; so that, the problem has codimension two.

The double bifurcation is encountered in several problems of practical interest, both in mechanical and structural engineering. In mechanics, an interesting example is given by a rigid, balanced isotropic rotor supported in air pressurized bearings and rotating about an elastically mounted rigid axis [2]. For special values of the angular speed and of a geometrical splitting parameter, a synchronous whirling motion and a self-excited vibration interact. This phenomenon also occurs in the motion of a tractor-semitrailer vehicle [3] controlled by the driving speed and the loading of the trailers, and in the dynamics of a pipe conveying fluid [4] and controlled by the flow rate and the static tension in the pipe. Within structural applications, both discrete and continuous models undergoing divergence-Hopf interaction, have been analyzed. Among the former a double pendulum has been studied, either loaded by a follower force and a radiant heat 
field [5], or subject only to a follower force, but with the stiffness spring varying as a splitting parameter [6]. Among the latter, the stability of a beam loaded by a follower force at the tip has been analyzed when the position of an intermediate support is varied [7].

The system behavior around a divergence-Hopf bifurcation point is far more complex than that around a single divergence or a Hopf bifurcation $[1,8]$, since the non-linear interactions between critical modes can produce interesting post-critical behaviors. From a computational point of view, and within the framework of the bifurcation theory [9], the problem is tackled by reducing the finite or infinite multidimensional dynamical system to an equivalent three dimensional system (i.e., to a dimension equal to the number of the critical eigenvalues occurring at bifurcation). In order to obtain the reduced system, the most commonly used approach is the center manifold method [8], which requires, first, a description of the manifold on which the post-critical steady state dynamics take place and, then, construction of the bifurcation equations in the so-called normal form. However, as observed by Nayfeh and Balachandran [10], this procedure entails a major computational effort for systems with large dimensions, since it is necessary to know both the complete spectrum of the Jacobian matrix at the critical state and the application of normal form theory.

Notwithstanding that normal form equations for low codimension bifurcations have been extensively studied in the literature, explicit expressions of the coefficients of the reduced system in terms of the coefficients of the original system are not available for general systems, so that the whole procedure has to be repeated for each specific problem. As already pointed out by the authors [11], other methods already used in the literature to solve static and dynamic bifurcation problems of codimension one can be used to analyze bifurcation of general systems, regardless of the codimension, and require less computational effort. In particular, the multiple scale method (MSM [12]) has been adapted to analyze the non-resonant Hopf-bifurcation of codimension two [11]. The reduced equations are obtained by the MSM without describing in advance the center manifold, expressing the Jacobian matrix at the critical state in Jordan form, or applying the normal form theory; in addition, stability is easily analyzed.

The aim of this paper is to show that the MSM can also conveniently be applied to general symmetric systems for which a divergence and a Hopf bifurcation interact. The presence of imperfections is also accounted for. An extension of the method to non-symmetric systems will be performed in a forthcoming paper, where some problems connected with the reconstitution procedure in the perturbative scheme [13,14] will be discussed. A strong formal analogy exists between the procedure illustrated here and that described in reference [11], apart from the imperfections, not accounted for there. In fact, the two approaches follow the same logic on which the static bifurcation theory of conservative systems is based [15]. A stronger analogy can also be observed, as the bifurcation equations are the same in the two problems.

A mechanical system consisting of a two-degree-of-freedom rigid bar (known as Augusti's model $[16,17])$ subjected to a fluid flow and to an axial load is studied. This structure was chosen as the simplest example to clarify the perturbative 
procedure while avoiding cumbersome algebra. However, in spite of its simplicity, the model exhibits all the fundamental aspects of the mechanical problem. Applications of the procedure to more complicated and realistic structures will be presented in a later paper.

\section{PROBLEM FORMULATION}

The equations of motion of an autonomous dynamical system affected by small imperfections are written in the form

$$
\dot{\mathbf{x}}=\mathbf{F}(\mathbf{x}, \boldsymbol{\gamma})+\mathbf{G}(\mathbf{x}, \boldsymbol{\gamma}, \boldsymbol{\eta})
$$

where $\mathbf{x}(t) \in \mathfrak{R}^{n}$ is the state vector, $\gamma \in \mathfrak{R}^{m}$ and $\boldsymbol{\eta} \in \mathfrak{R}^{\prime}$ are the control and imperfection parameter vectors, respectively; $\mathbf{F}$ is the vectorial field of the perfect system and $\mathbf{G}$ represents the (small) contribution of the imperfections to the system dynamics. For hypothesis $\mathbf{F}(\mathbf{0}, \gamma)=\mathbf{0} \forall \boldsymbol{\gamma}$ and $\mathbf{G}(\mathbf{x}, \gamma, \mathbf{0})=\mathbf{0} \forall(\mathbf{x}, \gamma)$. Therefore, when $\boldsymbol{\eta}=\mathbf{0}$, equation (1) admits the trivial equilibrium solution consisting of the set of states $\Gamma:=\{(\mathbf{x}, \gamma) \mid \mathbf{x}=\mathbf{0}\}$. According to Ljapunov's theory, such an equilibrium position is stable (or attracting) if each eigenvalue $\lambda_{i}(\gamma)$ of the Jacobian matrix

$$
\mathbf{F}_{\mathbf{x}}(\mathbf{0}, \boldsymbol{\gamma}):=\left.\frac{\partial \mathbf{F}(\mathbf{x}, \boldsymbol{\gamma})}{\partial \mathbf{x}}\right|_{\mathbf{x}=\mathbf{0}}
$$

has negative real part, while it is unstable if at least one of the eigenvalues has a positive real part [18]. The dynamical systems considered here depend on two control parameters, namely $\gamma=\{\mu, v\}^{\mathrm{T}}$, and one imperfection parameter $\eta$. The following hypotheses are assumed to hold for the perfect system.

(H.1) The dynamical system is symmetric, i.e.

$$
\mathbf{F}(-\mathbf{x}, \gamma)=-\mathbf{F}(\mathbf{x}, \gamma) \forall \gamma \text {. }
$$

(H.2) At the bifurcation point $\mathbf{O} \equiv(\mathbf{x}=\mathbf{0}, \mu=v=0)$ the Jacobian matrix $\mathbf{F}_{\mathbf{x}}^{0}:=\mathbf{F}_{\mathbf{x}}(\mathbf{x}=\mathbf{0}, \mu=0, v=0)$ has a null eigenvalue $\lambda_{0}=0$ and one pair of purely imaginary eigenvalues $\lambda_{1,2}= \pm i \omega_{0}$. The associated right eigenvectors $\mathbf{u}_{j}(j=0,1)$ are solutions of the following algebraic problems

$$
\mathbf{F}_{\mathbf{x}}^{\mathbf{0}} \mathbf{u}_{0}=\mathbf{0}, \quad \mathbf{F}_{\mathbf{x}}^{\mathbf{0}} \mathbf{u}_{1}=\mathrm{i} \omega_{0} \mathbf{u}_{1},
$$

while $\mathbf{u}_{2}=\tilde{\mathbf{u}}_{1}$, the associated left eigenvectors satisfy the equations

$$
\left(\mathbf{F}_{\mathrm{x}}^{0}\right)^{\mathrm{T}} \mathbf{v}_{0}=\mathbf{0}, \quad\left(\mathbf{F}_{\mathrm{x}}^{0}\right)^{\top} \mathbf{v}_{\mathbf{1}}=-\mathrm{i} \omega_{0} \mathbf{v}_{1},
$$

with $\mathbf{v}_{2}=\overline{\mathbf{v}}_{1}$. Right and left eigenvectors are orthonormal, i.e., $\mathbf{v}_{i}^{\mathrm{H}} \mathbf{u}_{j}=\delta_{i j}$, where $H$ denotes the transpose conjugate and $\delta_{i j}$ is the Kronecker symbol.

(H.3) At bifurcation, all the remaining eigenvalues $\lambda_{h}, h \geqslant 3$, lie on the left side of the complex plane.

(H.4) The critical eigenvalues

$$
\begin{gathered}
\lambda_{0}(\mu, v)=\alpha_{0}(\mu, v), \\
\lambda_{1.2}(\mu, v)=\alpha_{1}(\mu, v) \pm \mathrm{i} \omega(\mu, v), \\
\alpha_{j}(0,0)=0, \quad j=0,1,
\end{gathered}
$$


( $\mu$ and $v$ small) satisfy the transversality conditions

$$
\operatorname{det}\left[\begin{array}{ll}
\alpha_{0 \mu} & \alpha_{0 v} \\
\alpha_{1 \mu} & \alpha_{1 v}
\end{array}\right] \neq 0,
$$

where

$$
\alpha_{j \mu}:=\left.\frac{\partial \alpha_{j}}{\partial \mu}\right|_{\substack{\mu=0 \\ v=0}}, \quad \alpha_{j v}:=\left.\frac{\partial \alpha_{j}}{\partial v}\right|_{\substack{\mu=0 \\ v=0}} \text {. }
$$

In the parameter plane $(\mu, v)$ the curves $\alpha_{j}(\mu, v)=0, j=0,1$, determine the diagram of linear stability of the trivial solution $\Gamma$, also known as the stability boundary diagram. According to condition (7) the two curves have distinct tangents at the intersection point, where the codimension- 2 bifurcation takes place, so that no direction in the $(\mu, v)$-plane exists along which the critical state persists.

In the following, the multiple scale method is applied to analyze the postcritical behavior around the bifurcation point.

\section{THE MULTIPLE SCALE METHOD}

A monoparametric family of solutions of the type

$$
\begin{gathered}
\mathbf{x}=\mathbf{x}\left(\varepsilon, t_{0}, t_{2}, \ldots\right) \\
\mu=\mu(\varepsilon) \\
\nu=\nu(\varepsilon) \\
\eta=\eta(\varepsilon)
\end{gathered}
$$

is sought, in which $t_{0}=t, t_{2}=\left(\varepsilon^{2} / 2 !\right) t, \ldots, t_{2 k}=\left[\varepsilon^{2 k} /(2 k) !\right] t$ are independent temporal scales. Under hypotheses of regularity, equations (9) are expressed in MacLaurin series as

$$
\begin{gathered}
\mathbf{x}=\sum_{k=1,3, \ldots}^{\infty} \frac{\varepsilon^{k}}{k !} \mathbf{x}_{k}, \\
\left\{\begin{array}{l}
\mu \\
v
\end{array}\right\}=\sum_{k=2,4, \ldots}^{\infty} \frac{\varepsilon^{k}}{k !}\left\{\begin{array}{l}
\mu_{k} \\
v_{k}
\end{array}\right\}, \quad \eta=\sum_{k=3,5, \ldots}^{\infty} \frac{\varepsilon^{k}}{k !} \eta_{k},
\end{gathered}
$$

where $\mathbf{x}_{k}=\mathbf{x}_{k}\left(t_{0}, t_{2}, \ldots\right)$, and $\varepsilon=0$ selects the bifurcation point $\mathbf{O}$. It should be noted that, due to the symmetry property of the vector field (equation (3)), only odd or even powers of $\varepsilon$ are considered in the series (10) and (11a). In this way, if $(\mathbf{x}, \mu, v, 0)$ is a solution corresponding to $\varepsilon,(-\mathbf{x}, \mu, v, 0)$ is also a solution and corresponds to $-\varepsilon$. Moreover, only temporal scales of even order are taken into 
account, while the imperfection parameter is assumed to be smaller than the controls. The time derivative is expressed as

$$
\frac{\mathrm{d}}{\mathrm{d} t}=\mathrm{d}_{0}+\frac{\varepsilon^{2}}{2 !} \mathrm{d}_{2}+\cdots+\frac{\varepsilon^{2 k}}{(2 k) !} \mathrm{d}_{2 k}+\cdots
$$

where $d_{k}=\partial / \partial t_{k}$. By differentiating $k$ times equations (1) with respect to the perturbative parameter $\varepsilon$, evaluating the derivatives at $\varepsilon=0$ and using equations (10) and (11), the perturbative equations of $k$ th order are obtained; for $k=1,3$, they are

$$
\begin{gathered}
\left(\mathrm{d}_{0} \mathbf{E}-\mathbf{F}_{\mathbf{x}}^{0}\right) \mathbf{x}_{1}=\mathbf{0} \\
\left(\mathrm{d}_{0} \mathbf{E}-\mathbf{F}_{\mathbf{x}}^{0}\right) \mathbf{x}_{3}=3\left(\mu_{2} \mathbf{F}_{\mathbf{x} \mu}^{0}+v_{2} \mathbf{F}_{\mathbf{x} v}^{0}\right) \mathbf{x}_{1}+3 \mathbf{F}_{\mathbf{x x}}^{0} \mathbf{x}_{1} \mathbf{x}_{2}+\mathbf{F}_{\mathbf{x x x}}^{0} \mathbf{x}_{1}^{3}+\mathbf{G}_{\eta}^{0} \eta_{3}-3 \mathrm{~d}_{2} \mathbf{x}_{1} .
\end{gathered}
$$

It should be noted that, on account of the ordering (10) and (11), the generating equation (13a) is unaffected by imperfections; the behavior of the imperfect system is therefore evaluated as a perturbation of that of the perfect system.

The non-decaying solution of equation (13a) is

$$
\mathbf{x}_{1}=A_{0}\left(t_{2}, t_{4}, \ldots\right) \mathbf{u}_{0}+A_{1}\left(t_{2}, t_{4}, \ldots\right) \mathbf{u}_{1} \mathrm{e}^{\mathrm{i} \omega_{0} t_{0}}+c . c .,
$$

where $A_{0}=1 / 2 a_{0}\left(t_{2}, t_{4}, \ldots\right)$ is a real function, $A_{1}=1 / 2 a_{1}\left(t_{2}, t_{4}, \ldots\right)$

$\exp \left[\mathrm{i} \phi\left(t_{2}, t_{4}, \ldots\right)\right]$ is a complex function with real amplitude $a_{1}$ and phase $\phi$, and c.c. denotes the complex conjugate of preceding terms; both amplitudes are functions of slow time scales. Substitution of equation (14) in (13b) leads to

$$
\begin{aligned}
\left(\mathrm{d}_{0}-\mathbf{F}_{\mathbf{x}}^{0}\right) \mathbf{x}_{3} & =3\left[\left(-\mathrm{d}_{2}+\mu_{2} \mathbf{F}_{\mathbf{x} \mu}^{0}+v_{2} \mathbf{F}_{\mathbf{x} v}^{0}\right)+A_{1} A_{0} \mathbf{F}_{\mathbf{x x x}}^{0} \mathbf{u}_{1} \mathbf{u}_{0}+4 A_{0}^{2} \mathbf{F}_{\mathbf{x x x}}^{0} \mathbf{u}_{0}^{2}\right] A_{1} \mathbf{u}_{1} \mathrm{e}^{\mathrm{i} \omega_{0} t_{0}} \\
& +\left[3\left(-\mathrm{d}_{2}+\mu_{2} \mathbf{F}_{\mathbf{x} \mu}^{0}+v_{2} \mathbf{F}_{\mathbf{x v}}^{0}\right)+4 A_{0}^{2} \mathbf{F}_{\mathbf{x x x}}^{0} \mathbf{u}_{0}^{2}+6 A_{1} \bar{A}_{1} \mathbf{F}_{\mathbf{x x x}}^{0} \overline{\mathbf{u}}_{1} \mathbf{u}_{1}\right] A_{0} \mathbf{u}_{0} \\
& +\eta_{3} \mathbf{G}_{\eta}^{0}+\text { c.c. }+N S T
\end{aligned}
$$

where NST denotes non-secular terms. The solvability of equation (15) requires the coefficients of the resonant terms to be orthogonal to the left eigenvectors $\mathbf{v}_{0}$ and $\mathbf{v}_{1}$ of $\mathbf{F}_{\mathbf{x}}^{0}$ associated with critical eigenvalues 0 and $i \omega_{0}$, respectively, so that

$$
\begin{gathered}
\mathrm{d}_{2} A_{0}=\mathbf{v}_{0}^{\mathrm{T}}\left\{\left[\left(\mu_{2} \mathbf{F}_{\mathbf{x} \mu}^{0}+v_{2} \mathbf{F}_{\mathbf{x v}}^{0}\right)+\frac{4}{3} A_{0}^{2} \mathbf{F}_{\mathbf{x x x}}^{0} \mathbf{u}_{0}^{2}+2 A_{1} \bar{A}_{1} \mathbf{F}_{\mathbf{x x x}}^{0} \overline{\mathbf{u}}_{1} \mathbf{u}_{2}\right] A_{0} \mathbf{u}_{0}+\frac{1}{3} \eta_{3} \mathbf{G}_{\eta}^{0}\right\}, \\
\mathrm{d}_{2} A_{1}=\mathbf{v}_{1}^{\mathrm{H}}\left[\left(\mu_{2} \mathbf{F}_{\mathbf{x} \mu}^{0}+v_{2} \mathbf{F}_{\mathbf{x} v}^{0}\right)+A_{1} A_{0} \mathbf{F}_{\mathbf{x x x}}^{0} \mathbf{u}_{1} \mathbf{u}_{0}+4 A_{0}^{2} \mathbf{F}_{\mathbf{x x x}}^{0} \mathbf{u}_{0}^{2}\right] A_{1} \mathbf{u}_{1},
\end{gathered}
$$

By separating real and imaginary parts of the solvability conditions, the amplitude and phase modulation equations on the $t_{2}$-scale are drawn. By coming back to the $t$-scale and reabsorbing the parameter $\epsilon$, they are found to be

$$
\begin{gathered}
\dot{a}_{0}=\left(\alpha_{0 \mu} \mu+\alpha_{0 v} v\right) a_{0}+R_{110} a_{1}^{2} a_{0}+R_{000} a_{0}^{3}+R \eta+O\left(\left|a_{0}\right|^{5}+\left|a_{1}\right|^{5}\right) \\
\dot{a}_{1}=\left(\alpha_{1 \mu} \mu+\alpha_{1 v} v\right) a_{1}+R_{100} a_{0}^{2} a_{1}+R_{111} a_{1}^{3}+O\left(\left|a_{0}\right|^{5}+\left|a_{1}\right|^{5}\right)
\end{gathered}
$$

and

$$
\phi=\left(\omega_{1 \mu} \mu+\omega_{1 v} v\right)+I_{100} a_{0}^{2}+I_{111} a_{1}^{2}+O\left(\left|a_{1}\right|^{4}+\left|a_{2}\right|^{4}\right),
$$


where coefficients $\alpha_{j \mu}, \alpha_{j v}, \omega_{1 \mu}, \omega_{1 v}(j=1,2)$, given in the Appendix, have the meaning of partial derivatives of $\alpha_{j}$ and $\omega_{1}$ with respect to $\mu$ and $v$, evaluated at $\varepsilon=0$; coefficients $R$ 's and $I$ 's are also given in the Appendix.

The amplitude modulation equations (17) are uncoupled from the phase modulation equation (18) and can be studied, for example, by phase techniques. In the absence of imperfections, they constitute the bifurcation equations in standard normal form for one zero and one purely imaginary pair of critical eigenvalues for a symmetric system [19]. They are formally similar to amplitude modulation equations of the non-resonant double-Hopf bifurcation of a general two control parameter dynamical system [11]. Since they are invariant under the transformations $a_{0} \rightarrow-a_{0}$ and $a_{1} \rightarrow-a_{1}$, it is sufficient to consider positive only $a_{0}$ and $a_{1}$. When imperfections are taken into account, it is necessary to consider the positive half-plane $a_{1}>0$, only. Constant solutions of the equations (17) are determined by setting $\dot{a}_{0}=\dot{a}_{1}=0$. These solutions correspond to static solutions or periodic motions of the original system, equation (1). Equations (17) make it possible to detect the stability of periodic motions by analyzing the stability of equilibrium points. It is worthwhile observing that the imperfection term enters the equation in the buckling mode amplitude modulation only.

\section{A 2 d.o.f. SYSTEM UNDER COMPRESSIVE LOAD AND AERODYNAMIC EXCITATION}

In this section, the procedure described above is applied to the sample structure illustrated in Figure 1(a). It consists of a vertical rigid rod of length $l$ with a spherical hinge at the bottom end, restrained by two linear visco-elastic torsional hinges of stiffness $k_{1}$ and $k_{2}$, furnishing restoring moments proportional to the



(a)

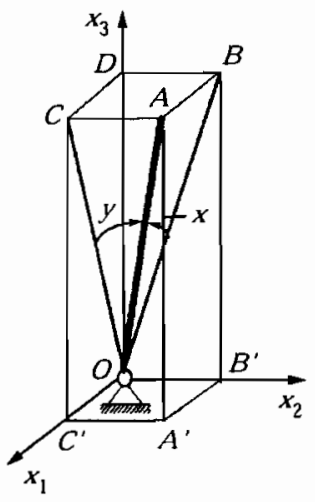

(b)

Figure 1. (a) 2-d.o.f rigid rod under compression load and aerodynamic excitation; (b) Lagrangian parameters. 
variations of the angles between the rigid rod axis and the $x_{1}$ - and $x_{2}$-axes respectively. The structure is loaded by a vertical force $P$ and is subject to a fluid flow of mean velocity $U$ acting in the positive direction of the $x_{1}$-axis. If only the vertical load is present, the system coincides with Augusti's model [16, 17], for which two different static bifurcations (buckling) occur for two critical load values. On the other hand, the fluid flow creates lift forces mainly in the $\left(x_{2}, x_{3}\right)$-plane that eventually lead to a Hopf bifurcation (galloping instability) if the rod cross-section is aerodynamically unstable. Due to the presence of both the vertical load and the fluid flow, the lower buckling mode and the galloping mode may interact.

Let $\alpha_{1}$ and $\alpha_{2}$ denote the angles between the direction of the rigid bar and the $x_{1}-$ and $x_{2}$-axes, respectively. The rotations $x:=\pi / 2-\alpha_{1}$ and $y:=\pi / 2-\alpha_{2}$, coincident with the strains of the springs, are taken as Lagrangian variables (Figure 1(b)). By applying the quasi-static theory for aerodynamic forces [20], under the hypothesis that the cross-section is symmetric with respect to the flow direction, the non-dimensional equations of motion, expanded up to the third order, are [21]

$$
\begin{gathered}
\ddot{x}+2\left(\xi_{s}+\xi_{d} u\right) \dot{x}+(1-p) x=-\frac{1}{6} p x^{3}+\frac{1}{2} p x y^{2}+\frac{3}{2} \eta u^{2} \\
\ddot{y}+2\left(\xi_{s}-\xi_{a} u\right) \dot{y}+(\beta-p) y=\frac{1}{2} p x^{2} y-\frac{1}{6} p y^{3}+\frac{c_{3}}{u} \dot{y}^{3}
\end{gathered} .
$$

In equations (19) $p$ and $u$ are non-dimensional control parameters, proportional to the vertical load and the fluid velocity, respectively; $\xi_{s}$ is the modal structural damping; $\xi_{d}>0$ and $\xi_{a}>0$ are the aerodynamical modal damping coefficients, depending on drag and lift forces; $\beta \gg 1$ is the ratio between the linear frequencies $\omega_{y}$ and $\omega_{x} ; \eta$ and $c_{3}$ are non-dimensional coefficients accounting for the effects of the fluid mean velocity and of the non-linear aerodynamic forces, respectively. Moreover, in equations (19) the dot denotes differentiation with respect to the non-dimensional time $\tau=\omega_{x} t$. In order to apply the theory developed above, equations (19) should be expressed in the form (1); however, as an example, the perturbative method will be applied directly to them.

When $\eta=0$, equations (19) admit the trivial solution $(x, y)=(0,0)$. Here $\eta$ is assumed to be small (i.e., it is assumed that the rod cross-section has a small drag coefficient), so that $\eta$ can be regarded as an imperfection parameter. In other words, from a physical point of view, the real system is considered to be obtained through a slight modification of an ideal perfect system on which no drag forces act.

In the perfect system, the trivial equilibrium position $(x, y)=(0,0)$ loses its stability through a divergence bifurcation when the modal stiffness in the $x$-direction vanishes; this occurs when $p=p_{c}:=1$, which triggers a buckling mode in the $\left(x_{1}, x_{3}\right)$-plane. Similarly, stability is lost through a Hopf bifurcation when the modal damping in the $y$-direction vanishes; this occurs when $u=u_{c}:=\xi_{s} / \xi_{a}$, which triggers a galloping mode in the $\left(x_{2}, x_{3}\right)$-plane.

The stability analysis of the trivial equilibrium position $x=y=0$ leads to the stability diagram in Figure 2, which shows the existence of two boundary stability curves. The curve $p=p_{c}$ corresponds to the static bifurcation and the curve $u=u_{c}$. 


$$
\dot{\phi}=-\frac{1}{2 \omega_{0}} \mu+\frac{1}{8 \omega_{0}} a_{0}^{2}+\frac{1}{16 \omega_{0}} a_{1}^{2}
$$

These equations are a particular case of the normal form equations (17) and (18), since some coefficients turn out to be identically zero. In the following the perfect system is analyzed first, then imperfections are accounted for.

\subsection{PERFECT STRUCTURE}

When $\eta=0$ equations (23) admit the trivial solution $a_{0 T}=a_{1 T}=0$. Non-trivial steady state solutions, with one or two non-vanishing components are sought. If $a_{1}=0$, equation (23b) is identically satisfied, while equation (23a) gives

$$
a_{0 B}^{2}=6 \mu \text {. }
$$

It describes the lower bifurcated branch of Augusti's model [16]. Similarly, if $a_{0}=0$, equation (23a) is identically satisfied, while equations (23b) and (24) yield

$$
a_{1 P}^{2}=-\frac{4}{3} \frac{u_{c} \xi_{a}}{c_{3} \omega_{0}^{2}} \nu, \quad \phi_{P}=-\frac{1}{2 \omega_{0}}\left(\mu+\frac{1}{6} \frac{u_{c} \xi_{a}}{c_{3} \omega_{0}^{2}} \nu\right) \tau+\phi_{0} .
$$

Equations (26) describe a periodic motion of amplitude $a_{1 P}$ and frequency $\phi_{P}$ subsequent to the Hopf bifurcation. Since $a_{1}$ is real, solution (26) exists only for certain ranges of the control parameters, depending on the sign of $c_{3}$. For example, if $c_{3}<0$, solution (26) exists only for $v>0$, while, if $c_{3}>0$, it exists only for $v<0$. Finally, if both $a_{0}$ and $a_{1}$ are different from zero (mixed solution), equation (23) gives

$$
a_{0 M}^{2}=2\left(-\frac{\xi_{a} u_{c}}{c_{3} \omega_{0}^{2}} \nu+3 \mu\right), \quad a_{1 M}^{2}=-\frac{4}{3} \frac{\xi_{a} u_{c}}{c_{3} \omega_{0}^{2}} \nu
$$

while the corresponding $\phi_{M}$ is obtained by direct substitution of equations (27) in equation (24). If $c_{3}<0$ the domain of definition of solution (27) is $v>0$ and $\left(\xi_{a} u_{c} /\left(\left|c_{3}\right| \omega_{0}^{2}\right)\right) v+3 \mu>0$. Since one of the two interacting modes is static, the resultant motion is periodic. From a mechanical point of view, the mixed solution corresponds to a periodic motion around a buckled (non-trivial) equilibrium position.

The stability analysis of the steady state solutions leads to the results represented in the bifurcation diagrams in Figure 3(a) (negative $c_{3}$ ) and Figure 3(b) (positive $c_{3}$ ). In these figures, phase-portraits are sketched for the different regions identified in the control parameter plane. The boundary lines for these regions are the axis $\mu, v$ and the line $r_{0}$ of equation $\mu=\left(\xi_{a} u_{c} / 3 c_{3} \omega_{0}^{2}\right) v$. It can be observed that, if $c_{3}<0$ (Figure 3(a)), stable periodic motions exist in region II (around the trivial equilibrium position) and in regions III and IV (around the buckled equilibrium position); a stable equilibrium position exists in region $V$. If $c_{3}>0$ (Figure 3(b)), stable post-critical periodic motions do not exist. The only stable equilibrium positions are the trivial solution (regions I, V) and the buckled solution (region IV). However, the attraction basins of these equilibrium positions do not fill the whole phase plane. 


\subsection{IMPERFECT STRUCTURE}

In order to evaluate the influence of the mean wind force, $\eta \neq 0$ is considered in equations (23). On inspecting equations (23), it is found that neither the trivial

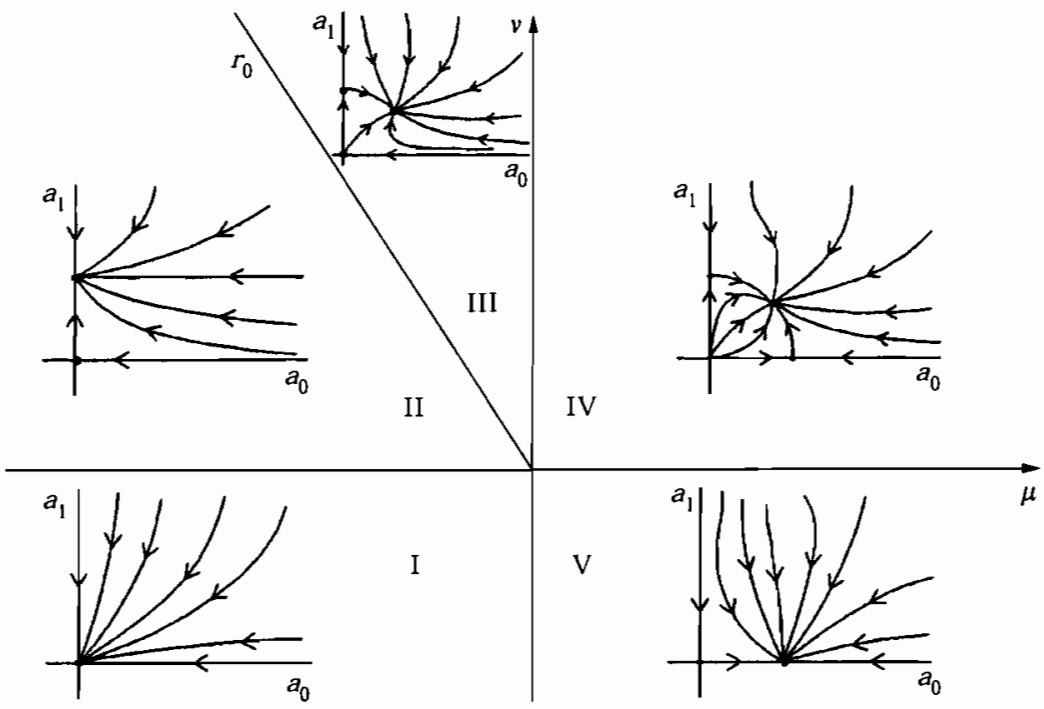

(a)
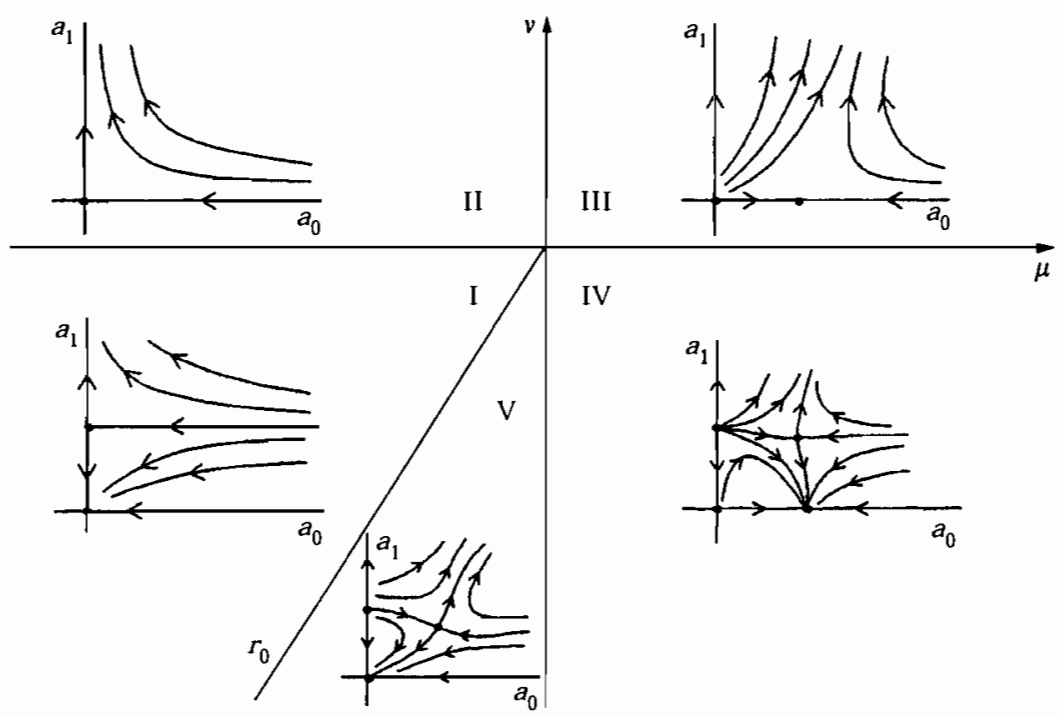

(b)

Figure 3. Bifurcation diagram in the $(\mu, v)$ parameter plane and phase portraits for the 2-d.o.f. perfect $(\eta=0)$ rigid model; (a) $c_{3}<0$, (b) $c_{3}>0$. 


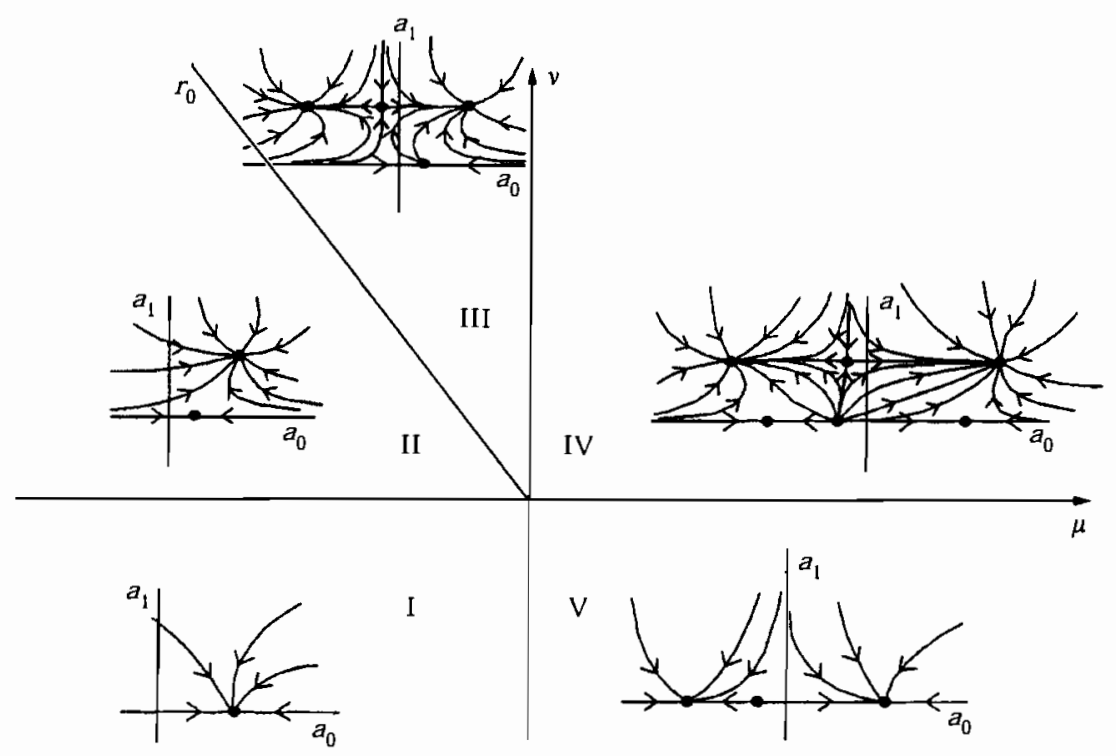

Figure 4. Bifurcation diagram in the $(\mu, v)$ parameter plane and phase portraits for the 2-d.o.f. imperfect $(\eta>0)$ rigid model $\left(c_{3}<0\right)$.

solution $\left(a_{0}=a_{1}=0\right)$ or the monomodal galloping $\left(a_{0}=0, a_{1} \neq 0\right)$ exist any longer. Static solutions $\left(a_{0} \neq 0, a_{1}=0\right)$ are furnished by the equation

$$
\mu a_{0}-\frac{1}{6} a_{0}^{3}+\frac{3}{4} \eta u_{c}^{2}=0,
$$

while mixed mode amplitudes $\left(a_{0} \neq 0, a_{1} \neq 0\right)$ are a solution of

$$
a_{0}\left(\mu-\frac{1}{3} \frac{\xi_{a} u_{c}}{c_{3} \omega_{0}^{2}} \nu\right)-\frac{1}{6} a_{0}^{3}+\frac{3}{4} \eta u_{c}^{2}=0,
$$

with $a_{1}$ still given by equation (27b). It should be noted, by comparing equations (28) and (29), that the amplitude $a_{1}$ produces an increase (decrease) in the linear stiffness in mixed modes if $c_{3}<0\left(c_{3}>0\right)$. The bifurcation diagram in the case $c_{3}<0$ is shown in Figure 4. Boundary lines are the same as those in Figure 3(a). From phase portraits, it is observed that imperfections destroy the symmetry with respect to the $a_{1}$-axis. Furthermore, one static solution exists for $\mu<0$, while three static solutions coexist for $\mu>0$, as is well-known from the buckling theory. In regions II, III and IV, stable periodic motions (around non-trivial equilibrium positions) exist. It is interesting to note that, as an effect of the interaction, a stable periodic motion exists in region III in which $a_{0}<0$, although no static solutions exist for $a_{0}<0$.

When $c_{3}>0$ (diagram not plotted), the boundary lines remain the same as those in Figure 3(b); static solutions are qualitatively the same as in case $c_{3}<0$, but stable periodic motions no longer exist. 


\section{CONCLUSIONS}

The Multiple Scale Method (MSM) has been used for the analysis of the post-critical behavior of non-conservative symmetric systems for which divergence and Hopf bifurcations occur simultaneously.

1. Closed form expressions for the coefficients of the bifurcation equations of a general system with two control and one imperfection parameters are obtained in terms of the coefficients of the original system. For practical purposes, they can be directly evaluated for each specific problem, without needing to repeat the whole procedure.

2. The proposed method is simpler than the commonly used center manifold and normal form method. Moreover, it is formally equal to the static perturbation method, which is more familiar to mechanical and structural engineers.

3. In the MSM, non-linear amplitude and phase modulation equations depending on the parameters $\mu, v$ and $\eta$, are obtained directly in normal form. To describe bifurcated paths as $a_{i}=a_{i}(\mu, v, \eta)$, non-linear equations in the amplitudes have to be solved. Stability analysis is then easily accomplished by using the same modulation equations.

4. The effect of small imperfections is easily accounted for in the algorithm. The solution is obtained as a perturbation of the solution of the perfect system.

5. The procedure was applied to an analysis of the post-critical behavior of a simple discrete structure. The example made it possible to highlight the main characteristics of the algorithm. However, this is believed to be a general and powerful method, whose potential usefulness should emerge particularly when applied to complex systems.

\section{REFERENCES}

1. W. F. LANGFoRd 1979 SIAM Journal of Applied Mathematics 37, 22-48. Periodic and steady-state mode interactions lead to tori.

2. A. ToNDL 1980 International Journal of Non-Linear Mechanics 15, 417-428. Determination of the limit of initiation of self-excited vibration of rotors.

3. V. Kacani, A. Stribersky, H. Troger and K. Zeman 1987 CMS-Conference Proceedings 8, 485-499. Dynamics and bifurcations in the motion of tractor-semitrailer vehicles.

4. P. J. Holmes 1977 Journal of Sound and Vibration 53, 471-503. Bifurcations to divergence and flutter in flow induced oscillations: a finite-dimensional analysis.

5. G. AUgusti 1968 Meccanica 3,1-10. Instability of struts subject to radiant heat.

6. R. SCheide, H. Troger and K. Zeman 1983 International Journal of Non-Linear Mechanics 19, 163-176. Coupled flutter and divergence bifurcation of a double pendulum.

7. I. Eishakoff and I. LotTati 1988 Computer Methods in Applied Mechanics and Engineering 66, 241-250. Divergence and flutter of nonconservative systems with intermediate support.

8. J. GUCKENHEIMER and P. Holmes 1983 Nonlinear Oscillations, Dynamical Systems and Bifurcations of Vector Fields. New York: Springer-Verlag.

9. V. I. ARNOLD 1982 Geometrical Methods in the Theory of Ordinary Differential Equations. Berlin: Springer-Verlag. (Russian original, Moscow, 1977).

10. A. H. NaYFeh and B. Balachandran 1995 Applied Nonlinear Dynamics. New York: Wiley-Interscience. 
11. A. Luongo and A. Paolone 1997 Nonlinear Dynamics 14, 193-210. Perturbation methods for bifurcation analysis from multiple nonresonant complex eigenvalues.

12. A. H. NAYFEH 1991 Introduction to Perturbation Techniques. New York: Wiley-Interscience.

13. Z. Rahman and T. D. BURTON 1989 Journal of Sound and Vibration, 133, 369-379. On higher order methods of multiple scales in non-linear oscillations-periodic steady state response.

14. A. Luongo and A. Paolone 1998 Report N. 4 DISAT, On the reconstitution problem in the multiple time scale method.

15. M. Pignataro, N. Rizzi and A. Luongo 1991 Stability, Bifurcation, and Postcritical Behavior of Elastic Systems. Amsterdam: Elsevier. (Italian first edition ESA, Rome, 1983).

16. G. Augusti 1964 Ph. D. Thesis, Department of Engineering, University of Cambridge. Some problems in structural instability, with special reference to beam-columns of I-section, part I: investigations on the basic types of elastic buckling and post-buckling by means of semi-rigid models.

17. G. Augusti 1964 Proceedings of the Physics and Mathematics Science of the Academy of Naples, Volume IV, Series $3^{\text {a }}$, N. 5 (in Italian). Stability of elastic structure in presence of large displacements.

18. V. I. ARNOLD 1973 Ordinary differential equations. Cambridge, MA: M.I.T. Press. (Russian original, Moscow, 1971).

19. H. TROGER and A. SteINDL 1991 Nonlinear Stability and Bifurcation Theory. Berlin: Springer-Verlag.

20. G. PICCARDo 1993 Journal of Wind Engineering Aerodynamics, 48, 241-252. A methodology for the study of coupled aeroelastic phenomena.

21. A. LuONGo and A. PAOLONE 1997 Proceedings of the XIII National Conference AIMETA, September (in Italian). Nonlinear interaction between quasi-simultaneous buckling and galloping modes.

\section{APPENDIX: COEFFICIENTS IN EQUATIONS (17) AND (18)}

By defining

$$
\begin{gathered}
\lambda_{j \mu}=\mathbf{v}_{j}^{H} \mathbf{F}_{\mathbf{x} j}^{0} \mathbf{u}_{j}, \quad \lambda_{j v}=\mathbf{v}_{j}^{H} \mathbf{F}_{\mathbf{x v}}^{0} \mathbf{u}_{j}, \\
c_{000}=\mathbf{v}_{0}^{T} \mathbf{F}_{\mathbf{x x x}}^{0} \mathbf{u}_{0}^{3}, \quad c_{111}=\mathbf{v}_{1}^{T} \mathbf{F}_{\mathbf{x x x}}^{0} \mathbf{u}_{1}^{2} \overline{\mathbf{u}}_{1}, \\
c_{110}=\mathbf{v}_{0}^{T} \mathbf{F}_{\mathbf{x x x}}^{0} \mathbf{u}_{0} \mathbf{u}_{1} \overline{\mathbf{u}}_{1}, \quad c_{100}=\mathbf{v}_{1}^{H} \mathbf{F}_{\mathbf{x x x}}^{0} \mathbf{u}_{1} \mathbf{u}_{0}^{2}, \quad c=\mathbf{v}_{0}^{T} \mathbf{G}_{\eta}^{0},
\end{gathered}
$$

the following equalities apply in equations (17) and (18)

$$
\begin{gathered}
\alpha_{j \mu}=\operatorname{Re} \lambda_{j \mu}, \quad \omega_{1 \mu}=\operatorname{Im} \lambda_{1 \mu}, \quad \alpha_{j v}=\operatorname{Re} \lambda_{j v}, \quad \omega_{1 v}=\operatorname{Re} \lambda_{1 v}, \\
R_{000}=\frac{1}{6} c_{000}, \quad R_{111}=\frac{1}{8} \operatorname{Re}\left(c_{111}\right), \quad R_{110}=\frac{1}{4} c_{110}, \quad R_{100}=\frac{1}{2} \operatorname{Re}\left(c_{100}\right), \\
R=\frac{2}{3} c, \quad I_{111}=\frac{1}{8} \operatorname{Im}\left(c_{111}\right), \quad I_{100}=\frac{1}{2} \operatorname{Im}\left(c_{100}\right) .
\end{gathered}
$$

